\title{
Die Letzte Eiszeit in Nordamerika und Europa
}

\author{
Von Paul Woldstedt, Bonn \\ Mit 8 Abbildungen im Text
}

\begin{abstract}
$\mathrm{Z}$ u s a m me $\mathrm{f}$ a s sung. Die Letzte Eiszeit (Würm/Wisconsin) zeigt in Nordamerika und Europa im großen denselben Verlauf. Das machen neue Beobachtungen und C14-Bestimmungen, besonders in den Randzonen der großen In'andeisgebiete, wahrscheinlich. Auf einen älteren, in sich gegliederten Eisvorstoß zwischen etwa 70 und $50000 \mathrm{~J}$. v. h. folgte zunächst ein kräftiges Interstadial (Göttweig, Talbot) und darauf ein mittlerer Abschnitt von wechselndem klimatischem Charakter, d. h. mit wärmeren Zeiten (Intervallen und Interstadialen), aber auch mit kälteren, in denen Lößbildung stattfand. Dieser Abschnitt reichte vom Ende des Göttweiger Interstadials (d. h. von rund 43000 bis etwa $25000 \mathrm{~J}$.v.h. In einem jüngeren Abschnitt fand ein erneuter, in sich wieder gegliederter Vorstoß statt, der fast überall zur Maximalausdehnung der Letzten Eiszeit führte und dann in Etappen zurückging. Er reichte von rund 25000 bis rund $10000 \mathrm{~J}$. v. h. Um diese Zeit ging die entscheidende Erwärmung vor sich, die die Letzte Eiszeit abschloß (wenn auch größere Eisreste diese Erwärmung zunächst noch überdauerten). Die drei Abschnitte der Letzten Eiszeit werden am besten als Alt-, Mittel- und Jungwürm bzw. Alt-, Mittel- und Jungwisconsin bezeichnet.
\end{abstract}

A bstract. As new observations and C14-determinations, especially in the surrounding of the big inlandices, show, the evolution of the Last Glaciation took place in the same manner in Europe as in North America. A first great ice advance (with minor oscillations) which lasted from about 70000 to about 50000 Years B. P., was followed first by a great interstadial (Göttweig, Talbot) and then by a middle part with a changing climatic character, viz. with warmer times (,interstadials") and colder ones (with formation of loess etc.). This part lasted from the end of the Göttweig-Interstadial (about 43000 ) to about 25000 Y.B.P. In the third part of the Last Glaciation a new great advance of the ice took place, which led to the maximum extension. Then the ice receded with some minor readvances. About 10000 Y.B.P. the temperature rose strongly and brought the Last Glaciation to an end (though some big ice relicts still existed).

The three parts of the Last Glaciation should be named: Old, Middle and Young Würm (Old, Middle, Young or Lower, Middle, Upper Wisconsin).

Eine große Anzahl von Arbeiten sowohl des deutschen wie des amerikanischen Schrifttums der letzten Jahrzehnte befaßt sich mit dem Ablauf der Letzten Eiszeit in den beiden Kontinenten (Würm, Wisconsin). Dieser Teil des Eiszeitalters ist der einzige, für den es bisher gelungen ist, mit Hilfe der $\mathrm{C}^{14}$-Methode absolute Zeitbestimmugnen zu erhalten und auf diese Weise ein wesentlich exakteres Bild vom Ablauf der Letzten Eiszeit zu gewinnen. Es liegt nahe, die Ergebnisse, wie sie sich für Amerika herausgestellt haben, mit denen in Europa zu vergleichen. ${ }^{1}$ )

\section{Das Letzte Interglazial (Eem, Sangamon)}

Es ist bisher nicht möglich gewesen, die Letzte Interglazialzeit (Eem, Sangamon) oder wenigstens ihr Ende exakt durch $\mathrm{C}^{14} \mathrm{zu}$ datieren. An der Gleichaltrigkeit des Eem- und Sangamon-Interglazials kann nicht gezweifelt werden (vgl. u. a. WoldSTEDT 1960, S. 15). Während der Ablauf des Eem-Interglazials, vor allem seiner Vegetations-Entwicklung, in weiten Teilen Europas wohl bekannt ist, läßt sich in Amerika bisher nur wenig über die Vegetations-Abfolge im Sangamon aussagen.

1) Auf zwei größeren Reisen (1928 und 1959), die beide von der Deutschen Forschungsgemeinschaft unterstützt wurden, war es mir möglich, die wichtigsten Gebiete Nordamerikas nicht nur im zentralen Vereisungsbereich, sondern auch in den Randgebieten im Osten, Süden und Westen des Kontinents kennen zu lernen. Ganz besonderen Dank schulde ich den zahlreichen amerikanischen Geologen, die mich in allen diesen Gebieten geführt haben. Es sind zuviele, als daß ich sie in einzelnen nennen könnte. 
In Europa zeigt sich über weite Gebiete eine außerordentliche Gleichmäßigkeit in der Vegetationsentwicklung der Gemäßigten Zone. Wohl stellen sich gewisse gesetzmäßige Anderung im Florenbild ein, wenn man von Westen nach Osten geht, so eine Zunahme der Linde und der Tanne, aber die Abfolge der Baum-Gemeinschaften bleibt eigentlich ïberall dieselbe, und es ist ohne weiteres möglich, ein Pollendiagramm von Westeuropa mit einem solchen aus Osteuropa zu vergleichen. Auch das nördliche Alpenvorland, aus dem bisher nur wenige Untersuchungen vorliegen - so die von Helga REICH (1953) über die Schieferkohlen von Großweil und Pfefferbichl - läßt sich ohne Schwierigkeit eingliedern.

Von einer solchen Übersicht sind wir in Nordamerika noch weit entfernt. Von Interglazialen, die wahrscheinlich in das Sangamon gehören, ist bisher nur das von T o r o n to pollenanalytisch untersucht worden (J. TERAsmaE 1959). Hier konnte nur ein Teil des Interglazials erfaßt werden, die „Don-Schichten“, die in der Hauptsache eine warme Phase (Temperatur-Mittel vielleicht $2-3^{\circ} \mathrm{C}$ wärmer als jetzt) und dann den Übergang zu einer kühleren Phase zeigen. Charakteristische Gipfel oder Baumgemeinschaften für bestimmte Abschnitte lassen sich noch nicht feststellen, da Vergleichsuntersuchungen aus anderen Gebieten fehlen. Wie ich an anderer Stelle schon hervorhob (WoLdSTEDT 1960), sollte es möglich sein, andere Sangamon-Interglaziale zu finden, am ehesten in alten Endmoränengebieten der Illinoian-Vereisung, und weiter diese Interglaziale zu verknüpfen mit den marinen Bildungen der Cape-May- oder Pamlico-Schichten. Dann könnte man auch in Nordamerika einen wichtigen Ausgangspunkt für weitere stratigraphische Untersuchungen gewinnen.

\section{Die Letzte Eiszeit in Nordamerika}

\section{a) Ostliches Nordamerika}

Uber den „Don-Schichten", die, wie wir sahen, mit großer Wahrscheinlichkeit in das Sangamon gehören, folgen, wie besonders A. P. Coleman (1933) nachwies, die „Scarborough-Schichten". Sie wurden bisher meist in einen kühleren Abschnitt der SangamonInterglazialzeit gestellt. J. TERASMAE (1959) hebt auf Grund seiner pollenanalytischen Untersuchungen hervor, daß die Scarborough-Beds wahrscheinlich mit einem subarktischen Klima beginnen und auch enden, während der Hauptteil borealen Charakter zeigt (Temperaturen etwa $5-6^{\circ} \mathrm{C}$ niedriger als heute). So scheinen die Scarborough-Beds also von dem Sangamon-Interglazial durch einen ersten Kältevorstoß getrennt zu sein, d. h. also ein (erstes ?) Intervall ${ }^{2}$ ) der Letzten Eiszeit zu bilden. Terasmae parallelisiert die Scarborough-Schichten mit dem St. P i e r r e - In t e r v a 11 in der Three Rivers-Gegend des St. Lorenz-Tieflandes (J. Terasmae 1957). Für dieses Intervall wurde nach A. DreIMANIS (1959) in Groningen ein Alter von rund $65000 \mathrm{C}^{14}$-Jahren vor heute ${ }^{3}$ ) ermittelt (Gro $1711=67000 \pm 1000$, Gro $1766=64000 \pm 2000)$. Es entspricht damit dem Amersfoort-Intervall in Europa.

2) G. LüTrig (1959) hat Vorschläge über die Begriffe „Interstadial“, „Interval'“ usw. gemacht. Sie erscheinen theoretisch richtig, sind aber in der Praxis nicht immer leicht anzuwenden. Wenn im folgenden "Interstadial" und "Intervall" unterschieden werden, so soll damit nur zum Ausdruck gebracht werden, daß in dem einen Falle („Interstadial“) eine vermutlich etwas länger dauernde, bedeutendere Erwärmung (mit entsprechendem Rückgang des Eises), im anderen Falle („Intervall“) eine kürzere, weniger bedeutende gemeint ist. Die Unterscheidung ist meist nur gefühlsmäßig.

3) Die Jahreszahlen werden von den Laboratorien meist als "Jahre vor heute" (d. h. vor dem Bestimmungstermin) angegeben. So sind sie auch hier zitiert worden. Die Vorgeschichtler sind dagegen gewohnt, in "Jahren vor (oder nach) Christus" zu rechnen. Man so!lte sich möglichst bald über eine einzige Rechnungsweise (eventuell mit neuem Nullpunkt) einigen, da immer wieder Verwechslungen der beiden Zählarten vorkommen. 


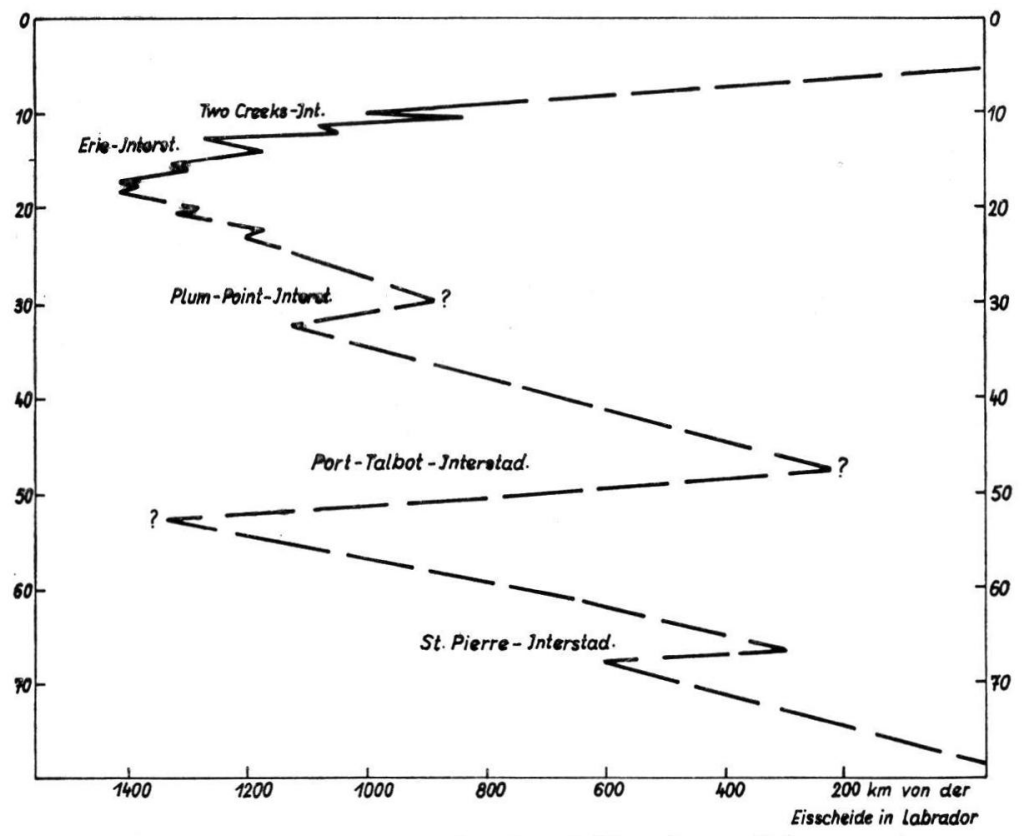

Abb. 1. Vereisungskurve (Letzte Eiszeit) für das Gebiet der östlichen Großen Seen und des St. Lorenz (nach A. Dreimanis 1959). Die Zahlen rechts und links bedeuten (hier und auf den anderen Abb.) Jahrtausende vor der Gegenwart.

Das Eis rückte dann (vgl. Abb. 1) zu einem ersten Maximum vor. ${ }^{4}$ ) Wo dieses Maximum erreicht wurde, ist noch umstrit .zn. Nach Dreimanis (1959) lag es jedenfalls südlich des Erie-Sees. Es folgte dann ein Rückzug („T albot-Interstadial“), der zum mindesten das Erie-Becken freigab. Denn in diesem wurain Beckenbildungen abgesetzt. Die palynologische Untersuchung eines Mehlsandes mit einer Gyttja zeigte, daß nach einer baumlosen Zeit Pinus, Picea, Larix und Betula einwanderten. Im Optimum waren Pinusformen mit großen Pollenkörnern vorhanden, die jedoch bald wieder verschwanden. Dieses Optimum ist nach Dreimanis mehr als $40000 \mathrm{C}^{14}$-Jahre alt. Der genannte Autor schätzt, daß das Ende des Talbot-Interstadials etwa bei 33-36 $000 \mathrm{C}^{14}$-Jahren v. h. liege. Der darauf folgende Eisvorstoß erfüllte wieder das Erie-Becken, ging aber wahrscheinlich nicht sehr weit darüber hinaus. Eine verhältnismäßig kurzfristige Erwärmung trieb den Gletscher wieder zurück, und Larix, Picea und wahrscheinlich auch Pinus wanderten für kurze Zeit ein. Es war das Plum-Point-Interstadia 1, das wahrscheinlich ein paar Jahrtausende dauerte und vielleicht um 25-28000 Jahre v. h. endete (C ${ }^{14}$-Daten: $\mathrm{L} 185 \mathrm{~B}=28200 \pm 1500$, W $177=27500 \pm 1200, \mathrm{~L} 217 \mathrm{~B}=24000 \pm 1600$ ).

Dann erst erfolgte nach Dreimanis der endgültige Vorstoß zum Maximum des „klassischen "Wisconsins, das bei rund 20-16000 J.v.h. erreicht wurde. Der Vormarsch

4) In Diskussionen über Vereisungs- und Klimakurven ist Kritik an der bisherigen sehr ungleichmäßigen Art der Kurvendarstellung geübt worden. Mal wird - bei horizontalen Kurven die Gegenwart links, mal rechts angenommen. Bei senkrechter Anordnung nimmt mal die Ausdehnung des Eises (oder die Abnahme der Temperatur) nach links zu, mal nach rechts. Es dürfte grundsätzlich richtig sein, die Kurven ähnlich anzuordnen wie Profile oder Pollendiagramme, d. h. daß das Alteste unten, das Jüngste oben erscheint. Es entsteht dann die Frage, ob man bei einer Klimakurve die negativen Werte links, die positiven rechts darstellen will oder umgekehrt. Ich möchte der erstgenannten Möglichkeit den Vorzug geben, und so sind die Klimakurven dieses Aufsatzes konstruiert worden. Für die Vereisungskurven ergibt sich dann, um sie mit den Klimakurven vergleichbar zu machen, daß man die Eisausdehnung von rechts nach links wachsen läßt. So sind jedenfalls die Vereisungskurven dieses Aufsatzes angeordnet. 
kann an zahlreichen Punkten im einzelnen festgelegt werden (vgl. u. a. R. F. FLINT 1957, S. 326; R. P. GoldthwaIt 1958). Dadurch daß eine zusammenhängende waldlose Tundrenzone vor dem Eisrande $\mathrm{nicht}$ existierte, sondern das Eis bei seinem Vorrücken in Waldland vorstieß, wurden vielfach Baumstämme in die Grundmoräne aufgenommen (vgl. hierzu G. W. Burns 1958). Sie gestatten meistens eine exakte Cl4-Bestimmung. So sind wir über den Vormarsch und den Rückzug des „klassischen "Wisconsins in Nordamerika besser orientiert als über den entsprechenden Abschnitt in Europa, wo vor dem Gletscher kein Waldland lag, sondern eine baumfreie Tundra.

Der Rückzug erfolgte ebenfalls in Schwankungen. Besonders bekannt ist das T w oCreeks-Interva11, das nach den $C^{14}$-Bestimmungen dem Alleröd-Intervall in Europa entspricht. Der darauf folgende Valders-Vorstoß muß dann den fennoskandischen Endmoränen („Salpausselkä-Phase“) entsprechen. Diese Gleichsetzung war zunächst etwas überraschend. Denn die Valders-Endmoränen liegen in Nordamerika noch durchaus im peripheren Bereich, während die Fennoskandischen Endmoränen bereits zum zentralen Vergletscherungsgebiet Europas gehören. Aber dieser Unterschied hängt offenbar mit der verschiedenen Größe der beiden Inlandeise zusammen. Das drei- bis viermal so große amerikanische Inlandeis reagierte im ganzen langsamer auf die spät- und postglaziale Erwärmung als das europäische (das grönlandische und das antarktische haben die holozäne Erwärmung überlebt).

Während sich nach A. Dreimanis (1959) im Gebiet des Erie-Sees zwischen den beiden Hauptvorstößen der Letzten Eiszeit zwei Interstadiale ausscheiden lassen, das längere Talbot-Interstadial und das kürzere Plum-Point-Interstadial, die durch einen kürzeren Eisvorstoß getrennt waren, verschmelzen die beiden Interstadiale im Randgebiet zu einem einzigen größeren, dem Sidney-Interstadial (vgl. hierzu u. a. Forsyth \& La Roque 1956, J. Forsyth 1958). Die Länge dieses Interstadials ist auf Grund der Verwitterungsschicht, die auf einem als Alt-Wisconsin angesehenen Geschiebemergel in Ohio entwickelt ist, auf etwa 16000 Jahre geschätzt worden. Das bedeutet aber nicht, $\mathrm{da}$ das eigentliche Interstadial so lange gedauert habe, sondern nur, daß dieses Gebiet in Ohio während etwa 16000 Jahren (vielleicht sogar noch wesentlich länger) eisfrei war. Das eigentliche Interstadial bzw. die eigentlichen Interstadiale machten nur einen Teil dieser Zeit aus.

Für den von den amerikanischen Quartärgeologen angenommenen Alt-WisconsinEisvorstoß liegen im übrigen eine ganze Reihe von $C^{14}$-Bestimmungen vor. Sie erscheinen aber fast alle in der Form: älte r als so und so viele Jahre, da die Laboratorien in Nordamerika ältere Werte nicht bestimmen konnten. So sind sichere Schlüsse aus diesen Werten nicht zu ziehen.

Ein weiterer Versuch, die Gliederung des Wisconsins graphisch darzustellen, ist jüngst von J. C. FrYe \& H. B. WILLMAN (1960) gemacht worden. Ihre Kurve wird in Abb. 2 wiedergegeben.

Auch hier werden zwei Hauptstadiale der Letzten Eiszeit unterschieden: ein älteres, hier als Altonian Substage bezeichnet, und ein jüngeres, das dem bisherigen „klassischen“ Wisconsin entspricht. Beide werden in dieser Darstellung durch das „F a r mda lian Substage " getrennt. Dieses soll von etwa 28000 bis 22000 Jahren v. h. gedauert haben.

Gegenüber der Darstellung von R. F. FLINT (1957, S. 326, die in Abb. 3 wiedergegeben wird) und der von A. Dreimanis (1959) ergeben sich einige Unterschiede. Die Datierung des „Farmdale-Interstadials“ (wie wir diesen Abschnitt bezeichnen würden) geht in der Hauptsache auf Holz zurück, das in Profilen nahe dem klassischen Farm-Creek-Profil (M. M. Leighton 1926) in entsprechender Position gefunden wurde. Die Werte sind: für eine Probe in den obersten $30 \mathrm{~cm}$ des Profils: $22900 \pm 900 \mathrm{~J} . \mathrm{v} . \mathrm{h}$. (W-68), für eine etwa $1 \mathrm{~m}$ tiefe Probe: $25100 \pm 800 \mathrm{~J}$. (W-69). Weitere Werte gehen bis $26100 \pm 600 \mathrm{~J}$. 


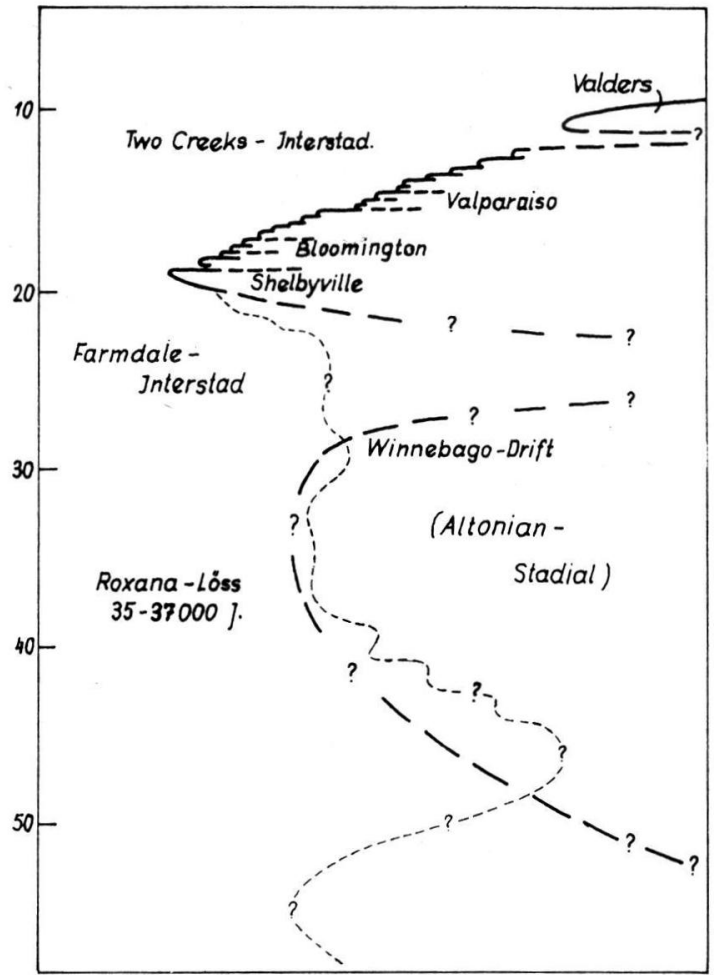

Abb. 2. V'creisungskurve (Letzte Eiszeit) für den Bereich des MichiganLobus (nach Frye \& Willman 1960). Mit gestrichelter Linie ist (von mir) eine andere mögliche Variante angedeutet worden.

(W-381). Der „Farmdale silt and peat" wurde also zwischen 23- und $26000 \mathrm{~J}$. v. h. gebildet. Darunter folgen $2-2^{1 / 2} \mathrm{~m}$ graubrauner Löß, der gelegentlich etwas humos und größtenteils entkalkt ist. Nur der tiefste halbe Meter zeigt noch Kalkgehalt. Darunter folgt die interglaziale Verwitterungsrinde des Illinoian-Geschiebemergels. Wie ich 1929 (S. 274) ausgeführt habe, muß der Löß, den Leighton später Farmdale-Löß genannt hat, in einer früheren Phase der auf das Sangamon folgenden Kaltzeit gebildet sein; ich vermutete damals, der Iowan-Vereisung. Die Boden- bzw. Torfbildung auf diesem Löß ist aber wesentlich jünger; sie hat sich, wie schon gesagt wurde, in der Hauptsache zwischen

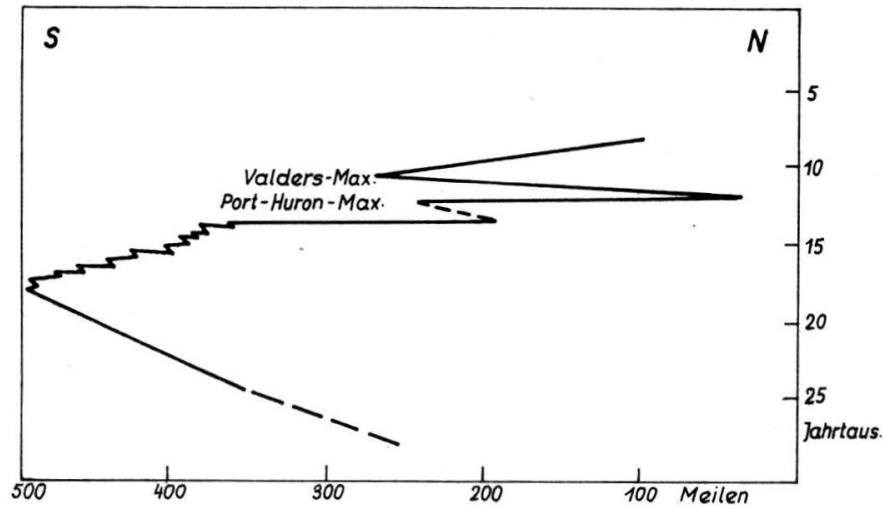

Abb. 3. Vereisungskurve des „klassischen" Wisconsins für das Gebiet der Großen Seen (nach R. F. FLINT 1957). 
23-26000 Jahren v. h. gebildet, was zeitlich etwa dem europäischen „Paudorf“-Horizont entsprechen würde (für das allerdings die bisherigen $\mathrm{C}^{14}$-Bestimmungen in Europa ein Alter von 25-28000 J.v. h. angeben). Dann setzte neue Lößbildung ein, offenbar im Zusammenhang mit dem Vorrücken des „klassischen Wisconsins“.

Die Kurve des Vorrückens des „klassischen“ Wisconsins ist von Frye \& WILlmaN (Abb. 2) reichlich steil gezeichnet worden, wesentlich steiler als der Rückzug bis zum TwoCreeks-Intervall. Ein so rasches Vorruicken des Inlandeises ist nicht sehr wahrscheinlich. Man wird eher ein etwas langsameres Vorrïcken erwarten. Es ist überhaupt die Frage, ob während des Farmdale-Interstadials ein sehr weites Zurückgehen des Eisrandes anzunehmen ist. Auf Abb. 2 ist eine andere Interpretation angedeutet worden, die unter Berïcksichtigung der sonstigen Ergebnisse möglich erscheint. Über das Alter des Lösses, auf dem sich der Farmdale-Boden und -Torf bildete, wissen wir mit Sicherheit nichts. Er könnte dem Mittleren Jüngeren Löß Europas entsprechen, könnte aber auch älter sein.

In der Tat wird von Frye \& W ILlman (1960) ein mächtiges älteres Paket Jüngeren Lösses in mehreren Profilen von Illinois angegeben. Es wird als „Roxana silt" bezeichnet. $\mathrm{C}^{14}$-Bestimmungen (auf Grund von Schneckenschalen) ergaben für Teile dieses Lösses $35200 \pm 1000 \mathrm{~J}$. (W-729) und $37000 \pm 1500 \mathrm{~J}$. (W-869) - Werte, die zu den neuen Altersbestimmungen von Oberfellabrunn (siehe unten) passen würden, ebenso auch zu der Kurve von A. Dreimanis (Abb. 1).

\section{b) Westliches Nordamerika}

Altersbestimmungen sind auch in den nicht vereisten Gebieten vorgenommen worden, so vor allem im Great Basin. Ganz im Südwesten dieses abflußlosen Gebietes liegt der heute völlig ausgetrocknete Searles-Lake. Die durch eine Reihe von Bohrungen aufgeschlossene Schichtenfolge des Sees zeigt nach R. F. FLINT \& W. A. GALE (1958) unterhalb von $67 \mathrm{~m}$ das sog. "Mixed layer“, das mindestens $200 \mathrm{~m}$ mächtig ist. Es repräsentiert wahrscheinlich eine Zeit, die klimatisch nicht allzu verschieden war von der gegenwärtigen. Es folgt der „Bottom Mud“, eine bis ca. $30 \mathrm{~m}$ mächtige Schicht von Ton, Silt und Salzen mit organischen Beimengungen. Sie repräsentiert eine feuchte Zeit (Pluvial), in der ein größerer See bestand, der seinerseits Zuflïsse erhielt und selber zum östlich gelegenen Panamint-Valley entwässerte. Nach Radiocarbon-Bestimmungen dauerte diese Zeit von $>46000$ J.v. h. bis $>32000$ J.v. h. Das Klima wurde wieder trockener, und Zu- und Abfluß des Sees hörten auf. Es wurde nun das 8-11 m mächtige Untere Salzlager abgesetzt, das eine trockene Zeit repräsentiert. Eine neue feinsandig-tonige Schicht („PartingMud"), 3-4 m mächtig, bezeichnet wieder eine feuchte Zeit, die nach Radiocarbon-Bestimmungen von $>23000$ bis etwa $10000 \mathrm{~J}$. v. h. dauerte. In der darauf folgenden trockenen Zeit wurde dann das obere Salzlager abgesetzt.

Vergleicht man diese Zeitbestimmungen mit den vorher besprochenen aus dem östlichen Nordamerika, so stimmt das Ende der Pluvialzeit im Westen ausgezeichnet überein mit dem Beginn der wärmeren Postglazialzeit im Osten. Auch der Anfang des letzten großen Eisvorstoßes im Osten stimmt einigermaßen mit den westlichen Zahlen überein, wenn man als Beginn des Vorstoßes das Ende des Farmdale-Interstadials ansieht. Geht man von den Kurven von Flint und Dreimanis aus, so müßte man den Beginn etwas früher ansetzen $(28-30000 \mathrm{~J}$. v. h.).

Ahnliche Untersuchungen wie am Searles-See sind von Broecker \& ORR (1958) für die beiden großen Seen des Great Basin, den Lake Bonneville und den Lake Lakontan, ausgeführt worden. Die C $C^{14}$-Bestimmungen beziehen sich hier auf Süßwasserkalke. Ihre Verläßlichkeit ist umstritten; doch soll der Fehler nach den genannten Autoren nicht mehr als $10 \%$ betragen. Beide Seen hatten nach diesen Untersuchungen einen Tiefstand, der von mindestens $34000 \mathrm{~J}$. v. h. an bestand. Es folgte (vgl. Abb. 4) ein Hochstand, der etwa von 25000 bis $14000 \mathrm{~J}$. v. h. dauerte. Bei ungefähr $13000 \mathrm{~J}$.v. h. hatten die Seen einen 

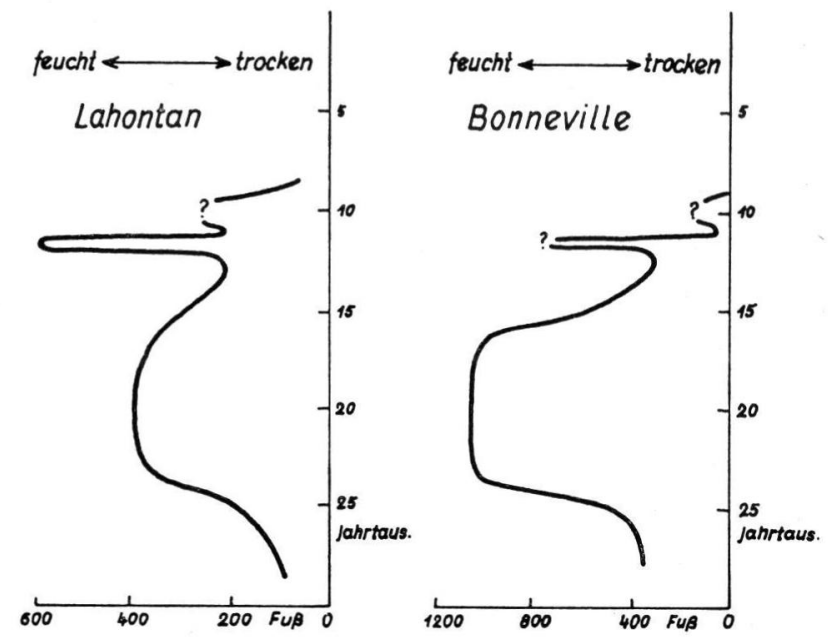
Abb. 4. Kurve der Wasser- stände im Lahontan- und Bonneville-See während des „klassischen“ Wisconsins (nach Broecker \& OrR 1958).

verhältnismäßig tiefen Stand, dem beim Lahontan (und vielleicht auch beim Bonneville) ein plötzlicher steiler Anstieg folgte; das Maximum wurde bei 11700 J.v.h. erreicht. Es folgte ein ebenso schnelles Absinken bis zu einem Minimum, das bei rund $11000 \mathrm{~J} . \mathrm{v} . \mathrm{h}$. lag. Ein letztes Maximum vor dem endgültigen Einschrumpfen hat möglicherweise gegen $10000 \mathrm{~J} . \mathrm{v} . \mathrm{h}$. bestanden.

Es liegt nahe, etwa folgende Parallelisierung durchzuführen:

Westl. Nordamerika

Tiefstand älter als 25000 J.v.h.

Hochstand zwischen 25000 u. 14000 J.v.h. Jung-Wisconsin

Tiefstand bei 13000 J.v.h.

Hochstand bei 11700 J.v.h.

Tiefstand bei 11000 J.v.h.

Hochstand bei 10000 J.v.h.
Ơstl. Nordamerika

Plum-Point-(Farmdale-) Interstadial

Wenig Übereinstimmung herrscht bisher bei den Werten für die vorhergehenden Abschnitte. Während im Osten eine Interstadialzeit etwa zwischen 50000 und 40000 J.v.h. und eine kürzere zwischen rund 30000 und 25000 Jahren angenommen wird, soll im Westen eine wärmere und trockenere Zeit zwischen $>32000$ und $>23000$ J.v.h. vorhanden gewesen sein. Die davor liegende kalte Zeit („Alt-Wisconsin“) lag im Osten zwischen etwa 70000 und etwa 50000 J.v.h., im Westen zwischen $>46000$ und $>32000$ J.v.h. Es wird weiterer $C^{14}$-Bestimmungen, besonders im Westen, bedürfen, bis man hier zur Klarheit kommt.

\section{Die Letzte Eiszeit (Würm, Weichsel) in Europa}

Nach den Untersuchungen von C. Emiliani (1958, S. 271) an Tiefsee-Bohrkernen endete die Letzte Interglazialzeit (R/W, Eem) vor etwa 70-80000 Jahren (Abb. 5). Wir haben dann eine Reihe von $\mathrm{C}^{14}$-Bestimmungen aus der älteren Würmzeit, die alle im Groninger C14-Laboratorium durch HI. DE VRIEs ausgeführt worden sind. Wenn auch die absoluten Werte dieser Bestimmungen wohl noch nicht über jeden $Z_{\text {weifel erhaben }}$ sind, so dürften sie doch größenordnungsmäßig richtig sein. Es erscheint möglich, danach den Verlauf der Letzten Eiszeit in Europa in großen Zügen zu rekonstruieren. 
Abb. 5. Temperaturkurve für die letzten 100000 Jahre, ermittelt an Tiefsee-Bohrkernen, und ihre Interpretation (nach C. Emiliani 1958).

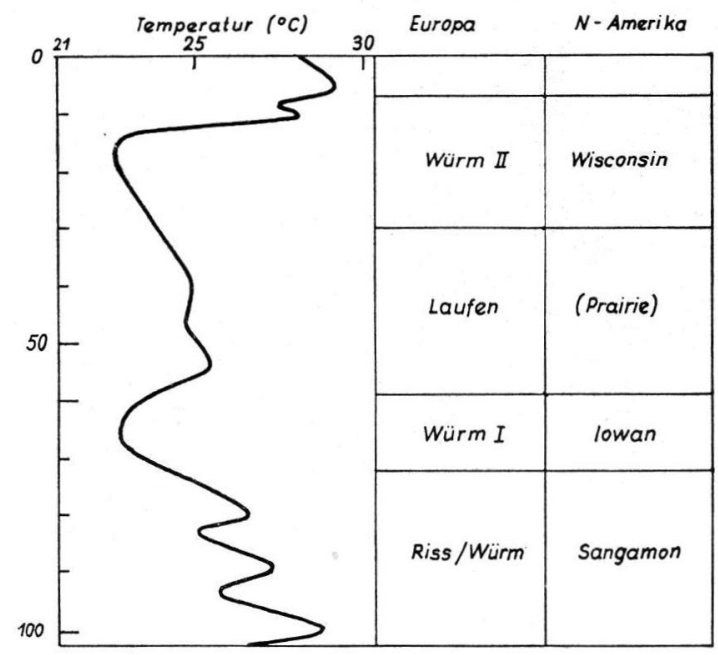

Ein ältester Wert ergibt sich für das im Hangenden der Eem-Schichten angetroffene Amersfoort-Intervall (nach dem Vorkommen Amersfoort XII), eine etwas wärmere Zeit nach einer ersten kühleren (Beginn von Würm). Es ist zu $64000 \pm 1100$ (Gro 1397) bestimmt (A. HARING u. a. 1958) und auch in Jütland (Rodebaek) festgestellt worden (vgl. Sv. Th. Andersen u. a. 1960). Ihm entspricht wohl, wie schon gesagt wurde, das St. PierreIntervall in Nordamerika. Ein zweites Interstadial (B r ö r u p) liegt bei etwa $59000 \mathrm{~J}$. (Gro $1470=59430 \pm 1000$, Gro $1729=58740 \pm 1000$; S. T. ANDERSEN u. a. 1960) und hat anscheinend mehrere Tausend Jahre gedauert. Es hat damit etwa dasselbe Alter wie die Pollenzone 11 (Picea-Dominanz) im Diagramm von Großweil-Ohlsdorf in Oberbayern (Helga REICH 1953). Auch in den Niederlanden ist es nachzuweisen. In dieselbe Zeit gehört vielleicht das Vorkommen von Chelford, Cheshire/England (etwas älter als 57000 J.), während die bekannte Moustier-Station von L ebenstedt (südl. Braunschweig) auf $55000 \pm 1000 \mathrm{~J}$. (Gro 2083) datiert wurde. Sie gehört damit wahrscheinlich wieder in eine etwas kältere Zeit.

Ob das Interstadial von L o o p s te d t bei Schleswig, dessen Ende nach Hl. DE VRIEs (1958) bei $50000 \pm 2000 \mathrm{~J}$. (Gro 1365) liegt, ein besonderes drittes Interstadial darstellt oder - was wahrscheinlicher ist - noch zum Brörup-Interstadial gehört, muß offen bleiben.

Über die Maximal-Ausdehnung dieses ersten Würm-Abschnittes ("Altwürm“) in Europa wissen wir bisher nichts. Es scheint, daß sie überall hinter der Maximal-Ausdehnung des Jungwürms zurückgeblieben ist. Etwa um 50000 J.v.h. begann das Eis wohl wieder zurückzugehen. Über diesen wie den dann folgenden mittleren Abschnitt der Letzten Eiszeit wissen wir bisher sehr wenig. Andersen, DE VRIEs \& $Z_{\text {AGwiJN (1960) glauben }}$ in der langen Zeit zwischen etwa 55000 und 14000 J.v.h. überhaupt nur eine etwas wärmere Schwankung feststellen zu können (bei etwa 28-30000 J.), die sie als möglicherweise gleichaltrig mit Paudorf ansehen. Die Dinge liegen jedoch offenbar viel komplizierter.

Dies zeigt auch eine Kurve, die Arlette Leror-Gourhan 1959 veröffentlicht hat (leider noch ohne Text) und die hier in etwas vereinfachter Form wiedergegeben wird (Abb. 6). Sie zeigt in dem uns hier interessierenden Abschnitt unterhalb von 29000 J.v.h. zunächst ein kürzer dauerndes, aber ausgeprägtes Interstadial, das als Arcy-Interstadial bezeichnet wird. In sie werden die Aurignac-Vorkommen von Arcy-sur-Cure, aber auch 


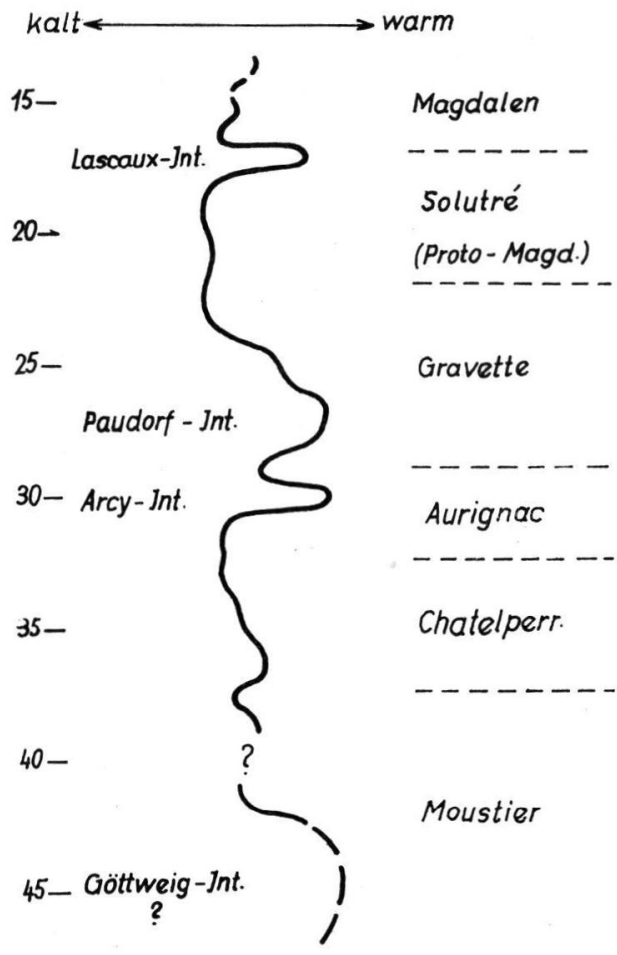

Abb. 6. Temperaturkurve für Frankreich während der Zeit von etwa $48000-12000$ Jahren vor der Gegenwart (nach A. LeroIGourhan 1959).

die von Istállóskő (Ungarn) und La Quina gestellt. Davor soll eine kalte Zeit von einigen Tausend Jahren liegen und davor wieder eine etwas wärmere, in die der Hauptteil des Châtelperrons gestellt wird. Das eigentliche Göttweiger Interstadial wird von A. LeroIGourhan zwischen etwa 42 und 47000 J.v.h. vermutet. Die Kurve basiert hauptsächlich auf zahlreichen Pollen-Untersuchungen, die die Verfasserin besonders in der Schichtenfolge von Arcy-sur-Cure, aber auch an anderen Stellen ausgeführt hat, und auf einer Reihe von $\mathrm{C}^{14}$-Bestimmungen (vgl. für diesen Abschnitt und das Jungwürm auch $\mathrm{H}$. L. Movius 1960, H. Gross 1958, 1959, 1960 sowie J. NARR 1959, 1960).

Wenn wir zu einer geologischen Beurteilung dieses Abschnittes der Letzten Eiszeit kommen wollen, müssen wir noch etwas weiter ausgreifen. Wie die Untersuchungen der letzten Jahrzehnte gezeigt haben, läßt der Würm-Löß, seit langem, besonders seit W. SoERgeL's grundlegenden Untersuchungen (1919), als "Jüngerer Löß“ bezeichnet, eine Gliederung in 3-4 Abschnitte erkennen. So unterscheidet F. Bordes (1954) im SeineBecken oberhalb des "argile rouge“, der die Verwitterungszone des Eem-Interglazials darstellt, drei Jüngere Lösse, die durch fossile Böden getrennt sind. Die beiden unteren Jüngeren Lösse enthalten Moustier-Kulturen, während sich im oberen Jungpaläolithikum (Périgord, Aurignac usw.) findet. In Mitteleuropa (vgl. u. a. E. Schönhals 1951, F. Brandtner 1954, 1956, Prošek \& Ložek 1957, H. Remy 1960) ist ebenfalls eine Dreigliederung des Jüngeren Lösses festgestellt worden. Doch deckt sich diese Gliederung nicht mit der in Frankreich aufgestellten. Der untere Jüngere Löß in Mitteleuropa, der hier die (seltenen!) Moustier-Kulturen enthält, entspricht offenbar dem Jüngeren Löß I und II des Seine-Beckens, und die Jüngeren Lösse II und III Mitteleuropas mit jungpaläolithischen Kulturen (Aurignac, Gravette usw.) sind die Åquivalente des (oftmals zweigeteilten) Jüngeren Lösses III des Seine-Beckens (vgl. hierzu F. Bondes 1958). Es ergibt sich also, wie ich 1956 ausgeführt habe, folgende Gleichstellung: 


$$
\begin{aligned}
& \text { Frankreich } \\
& \left.\begin{array}{l}
\text { IIIb } \\
\text { IIIa }
\end{array}\right\} \begin{array}{l}
\text { Solutré z. T. } \\
\text { Aurignac } \\
+ \text { Périgord }
\end{array} \\
& \left.\begin{array}{r}
\text { II } \\
\text { I }
\end{array}\right\} \text { Moustier }
\end{aligned}
$$

Mitteleuropa

$$
\left.\begin{array}{r}
\text { III } \\
\text { II }
\end{array}\right\} \begin{aligned}
& \begin{array}{l}
\text { Gravette } \\
\text { Aurignac } \\
+ \text { Szeleta }
\end{array} \\
& \text { I } \\
& \text { Moustier }
\end{aligned}
$$

Es scheint, daß auch der Jüngere Löß I von Mitteleuropa öfters zweigeteilt ist; zum mindesten trifft dies für das Niederrheingebiet $\mathrm{zu}$, wie neuere, noch im Gange befindliche Untersuchungen gezeigt haben (vgl. u. a. H. REMY 1960). So ist also eine weitgehende Übereinstimmung zwischen Mittel- und Westeuropa vorhanden. Die einzelnen Stufen des Jüngeren Lösses werden durch mehr oder weniger entwickelte Böden getrennt, die sich regional verfolgen lassen. Zwischen den mitteleuropäischen Jüngeren Lössen I und II ist eine Bodenbildung besonderer Art entwickelt, die F. Brandtner (1954, S. 73) als „F e llabrunner Bodenbildungskomplex" bezeichnet hat. Der eigentliche interstadiale Boden wird durch eine Verlehmung bezeichnet, während darüber ein mehrfacher Wechsel von Löß- und Schwarzerdebildung vorhanden ist (vgl. Abb. 6 bei BranntNer 1954). Dieses Abwechseln von Löß und Schwarzerde bezeichnet den Übergang vom Interstadial zum II. Würmstadial. Ofienbar fand hier ein rascher Wechsel zwischen etwas kälteren Löß- und etwas wärmeren Schwarzerde-Bildungszeiten statt, wie ich dies in meiner Kurve der Letzten Eiszeit (Woldsted 1958a, 1958b, S. 244) angedeutet habe (vgl. auch Abb. 8).

Für das eigentliche Interstadial, dessen Bodenbildung im unteren Teil des Profils Fellabrunn (Verlehmungszone) vorliegt, ist seit J. BAYER (1913) die Bezeichnung "Göttweiger Interstadial“ gebraucht worden. F. Zeuner (1954) und H. Gross (1956) haben sie wieder aufgenommen, und ich bin dieser Namensgebung gefolgt (1958b, S. 237), halte sie aber heute doch nicht für sehr glücklich. Denn gerade in Göttweig selber ist der sichere Nachweis nicht zu erbringen, daß der dort ausgebildete Boden wirklich in den Jüngeren Löß gehört. So hätte man vielleicht besser den von BRANDTNER vorgeschlagenen Namen: „Fellabrunner Bodenbildungskomplex" anwenden sollen. Aber die Bezeichnung "Göttweiger Interstadial“ spielt seit langem in der Literatur eine Rolle, und so sei sie in dieser Arbeit weiter verwendet. H. GRoss (dieser Band S. 100) versteht neuerdings darunter den ganzen länger dauernden Komplex, der die Letzte Eiszeit in zwei Hauptabschnitte teilt.

Die entscheidenden Fragen sind nun die: wann war dieses Interstadial bzw. dieser „Interstadial-Komplex"? Und wie ist er klimatisch zu beurteilen? Leider ist in Mitteleuropa bisher ein ganz klares Bild noch nicht zu gewinnen.

Zunächst ist hier die $\mathrm{C}^{14}$-Bestimmung von Holzkohle aus der Hauptfundschicht der Höhlenbärenreste in der Salz of enhöhle (Elisabeth Schmid 1958) zu besprechen. Sie hat ein Alter von $34000 \pm 3000 \mathrm{~J}$. (Gro-761) ergeben. Die Salzofenhöhle liegt in $2008 \mathrm{~m}$ ü.d.M. Nun sollte man sich auf eine solche einzelne $\mathrm{C}^{14}$-Bestimmung nicht allzusehr verlassen. Man sollte sie vor allen Dingen so schnell wie möglich durch weitere Bestimmungen ergänzen. Aber wenn die Bestimmung nicht überhaupt völlig falsch ist und die Schicht möglicherweise in das $\mathrm{R}$ /W-Interglazial gehört, wird man soviel sagen können: zu irgend einer Zeit des mittleren Würms muß die Vergletscherung der Alpen soweit zurückgegangen sein, daß eine Begehung der Salzofenhöhle möglich war. $\mathrm{Ob}$ das dieselbe Zeit war, als die in $1700 \mathrm{~m}$ Höhe in den Karawanken gelegene Potočka-Höhle mit ihrer Olschewa-Kultur (vgl. u. a. S. Brodar 1938) begehbar war, ist nicht gesagt. Die Begehung der Potočka-Höhle könnte auch in dem von A. LeroI-Gourhan angenommenen, späteren Arcy-Interstadial stattgefunden haben.

Wenn Höhlen in 2000 und $1700 \mathrm{~m}$ Höhe begehbar waren, so müssen wir auf jeden Fall mit einer kräftigen Erwärmung und einem starken Rückgang des Eises in den Alpen 


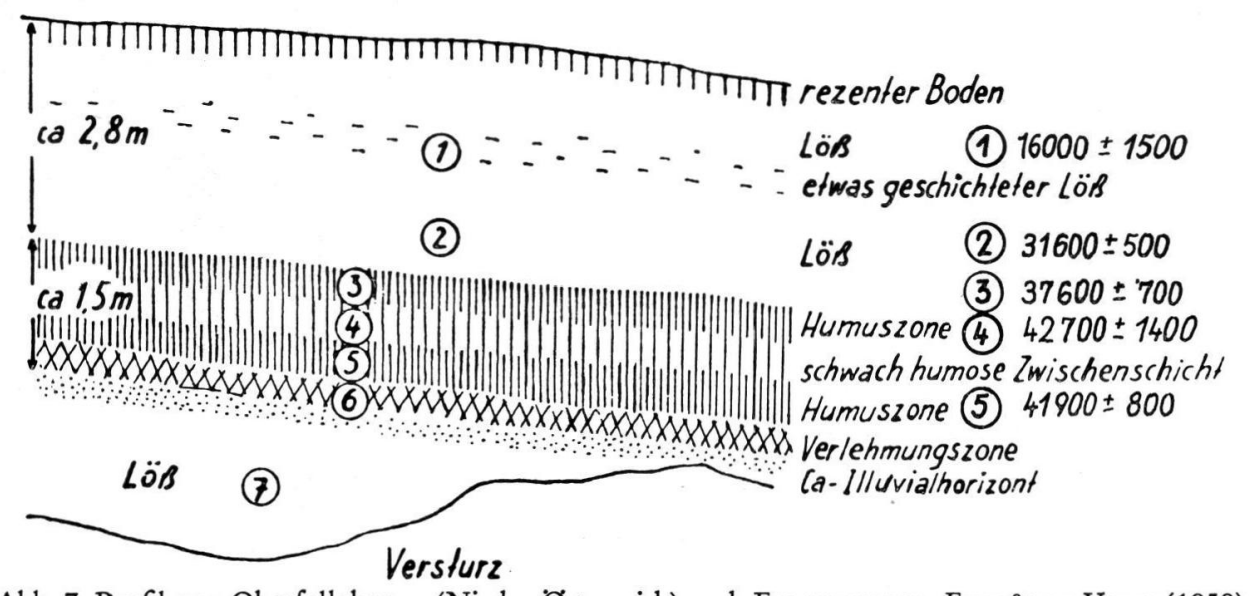

Abb. 7. Profil von Oberfellabrunn (Nieder-Ósterreich) nach Felgenhauer, FinK \& DE VRIEs (1959) mit den für die Schichten $1-5$ ermittelten C14-Alterszahlen (Jahre vor heute).

rechnen. Es entzieht sich aber jeglicher Schätzung, wie weit etwa das Nordische Inlandeis zurïckgegangen war. -

Weiter sind die neuen $\mathrm{C}^{14}$-Bestimmungen zu erörtern, die für das Profil von Oberfellabrunn ausgeführt worden sind (Felgenhauer, Fink \& DE VRIEs 1959). Der eigentliche Verlehmungshorizont, der mit dem Göttweiger parallelisiert wird, ist hier, wie schon oben auseinandergesetzt wurde, überlagert von mehreren Humuszonen, die mit der Verlehmung nichts zu tun haben, sondern jüngere Bodenbildungen darstellen. In Groningen ausgeführte $\mathrm{C}^{14}$-Bestimmungen lieferten die in $\mathrm{Abb} .7$ angegebenen Werte. Es erscheint etwas überraschend, daß zwischen den beiden im Löß genommenen Proben 1 und 2 ein Zeitraum von beinahe 16000 Jahren (!) liegen soll. Das ist an sich wohl möglich, aber doch nicht gerade zu erwarten. In 6000 Jahren Abstand folgt die Probe 3 in der oberen Humuszone, in weiteren 5000 Jahren Probe 4 in der schwach humosen Zwischenschicht, während für die in der unteren Humuszone entnommene Probe 5 wieder ein etwas geringeres Alter als für 4 angegeben wird. Offenbar liegen hier noch Unsicherheiten in der Bestimmung vor, so daß einstweilen Vorsicht geboten ist. Sollte der Wert von rund 42000 für die untere Humuszone richtig sein, so ergäbe sich für die darunterliegende Verlehmungszone, die ja im allgemeinen mit dem Göttweiger Interstadial gleichgesetzt wird, ein noch größeres Alter. Sie müßte dann etwa mit dem Torfband von $\mathrm{Hörmating}$ (45000 J.) gleichaltrig sein. Das von Edith EBErs in diesem Band beschriebene Profil von Hörmating in Oberbayern wird im einzelnen von $\mathrm{H}$. Gross mit dem Profil von Oberfellabrunn verglichen (dieser Band, S. 99 bis 106).

Über die klimatische Entwicklung des Göttweiger Interstadials im engeren Sinne (Hörmatinger Interstadial) wissen wir bisher sehr wenig. Wenn es zutrifft, daß der „obere Torf“ von Ci mos z k ow i c z e bei Nowogrodek, den M. GawŁowsKa (1934) bearbeitet hat, in das Göttweiger Interstadial gehört, dann würde das Pollendiagramm (vgl. WoLDSTEDT 1958b, Abb. 45 auf S. 130) einen Anhalt für die Klimaentwicklung geben. Beherrschende Bäume sind Pinus, Betula und Alnus. Picea, Eichenmischwald und Corylus sind in geringen Prozenten vertreten. Wir müssen dabei bedenken, daß es sich hier um ein kontinentales, sommerwarmes Gebiet handelt. Im Westen werden wir mit Laubbäumen nur an besonders begünstigten OOrtlichkeiten rechnen können. Es sollte versucht werden, von Cimoszkowicze so bald wie möglich eine $\mathrm{C}^{14}$-Bestimmung zu bekommen.

Auf das eigentliche Göttweiger (=Hörmatinger) Interstadial folgte der schon geschilderte rasche Wechsel zwischen Lößaufwehung und Schwarzerde-Bildung. Aber es 
muß auch in der darauffolgenden Zeit des Mittelwürms noch zu weiterer Lößbildung gekommen sein, wie u. a. die neuen Bestimmungen von Oberfellabrunn zeigen.

Die Kulturschichten 4 und 5 der Station Willendorf II (Felgenhauer, Fink \& DE VRIEs 1959, S. 37) haben ein Alter von etwa 32000 J. ergeben (Schicht 4 mit Aurignac s. str.: Gro $1273=31840 \pm 2500$ J., Schicht 5: $32000 \pm 3000 \mathrm{~J}$.). Sie sollen nach Felgenhauer kaltzeitlich sein und würden, wenn man die Kürve von A. Leroi-Gourhan zugrunde legt, in die Zeit v or das Arcy-Interstadial gehören. Von einem solchen ArcyInterstadial ist allerdings im Profil von Willendorf nichts zu sehen.

Immerhin müssen wir nach den Pollen-Untersuchungen von A. LeroI-Gourhan mit dem Auftreten dieses Interstadials rechnen, das in seiner Zeitstellung etwa dem PlumPoint-Interstadial Nordamerikas entsprechen würde.

Es entsteht dann die Frage, ob man hierher vielleicht auch die Schieferkohlen im Glütschtal mit einem Alter von $29000 \pm 1500$ J. (Oeschger u. a. 1959), ferner Torfe vom Karrestobel mit einem fast gleichen Alter (Gro $1260=29000 \pm 500$, Gro $1277=$ $28840 \pm 300$ ) stellen könnte.

Bei ungefähr 25-28000 J.v.h. liegt dann das bekannte Paudorfer Interstadia l. Holzkohle aus Gravette-Schichten unmittelbar über dem fossilen Boden ergab für das Vorkommen von Pollau (Pavlow) in Mähren ein Alter von 25-26000 Jahren (Gro $1272=26400 \pm 230 \mathrm{~J}$; ; Gro $1325=24800 \pm 150 \mathrm{~J}$.), für ein entsprechendes Vorkommen von Unterwisternitz (Dolni Vestonice), Mähren, ein Alter von $25600 \pm 170 \mathrm{~J}$. (Gro 1286). Das Ende der Paudorfer Schwankung mag also bei 25-26000 J.v.h. gelegen haben, und dann begann der Vorstoß zur Maximalausdehnung der Würm-Vereisung.

In Norddeutschland wurde vom Eise wahrscheinlich erst die (später überfahrene) Stettiner, dann die Brandenburger und Frankfurter Eisrandlage erreicht. Wann dies der Fall war, wissen wir nicht genau, da $\mathrm{C}^{14}$-Bestimmungen für diese Endmoränen fehlen. Für die Pommersche Endmoräne kann ein Mindestalter von etwa 16000 Jahren v. h. (14 000 J.v.Chr.) angenommen werden (vgl. WoldstedT 1958b, S. 151), für das Brandenburger. vielleicht ein solches von 18000 J.v.h. Wir kommen damit auf ganz ähnliche Zahlen, wie sie für das Maximum des Jung-Wisconsins in Nordamerika durch $\mathrm{C}^{14}-\mathrm{Be}-$ stimmungen festgelegt worden sind.

Es kann keinem Zweifel unterliegen, daß auch die alpine Würm-Vereisung ihr Maximum in derselben Zeit erreichte. Die teilweise als „Würm I“, „Würm II“ und „Würm III“ bezeichneten Endmoränen gehören alle in das Jungwürm und liegen zeitlich offenbar ganz nahe zusammen.

Der Rückzug des Eises vom Maximum bis zur Postglazialzeit ist bekannt genug, so daß er hier nicht nochmals behandelt zu werden braucht.

In Abb. 8 habe ich versucht, auf Grund der hier gemachten Ausführungen eine neue Kurve zu zeichnen. Dabei ist außer meinen eigenen früheren Kurven (zuletzt 1958) auch die Kurve von H. Gross (1958) mit verwendet worden. Auf Grund dieser sowie der andern in diesem Aufsatz behandelten Kurven ergibt sich eine Gliederung der Letzten Eiszeit in 3 Hauptabschnitte: einen älteren Hauptvorstoß, eine mittlere Zeit von wechselndem klimatischem Charakter und einen jüngeren Hauptvorstoß. Logischerweise wird man diese 3 Abschnitte der Würm-Eiszeit als Alt-, Mittel- und Jungwürm bezeichnen müssen. Die bisherigen Bezeichnungen $\mathrm{Alt}$ - (oder Früh-) und $\mathrm{H}$ a u ptw ü rm (Ed. Ebers 1955, H. Gross 1958) sind nicht befriedigend (denn "Alt" und „Haupt“ sind kein logisches Begriffspaar). Aber auch die von mir verwendeten: $\mathrm{F} \mathrm{r}$ ü h - , $\mathrm{Mitte} 1$ - und $\mathrm{Spätw}$ ürm treffen nicht das Wesentliche. Ich war ausgegangen von dem seit Jahrzehnten in der Literatur verwendeten Begriff: „Spätglazial“ und wollte ihn durch „Spätwürm“ ersetzen. Daraus ergab sich dann die Ausscheidung eines „Früh“und „Mittel-Würms“, wobei das Mittelwürm die ganze Zeit vom Beginn des Göttweiger 


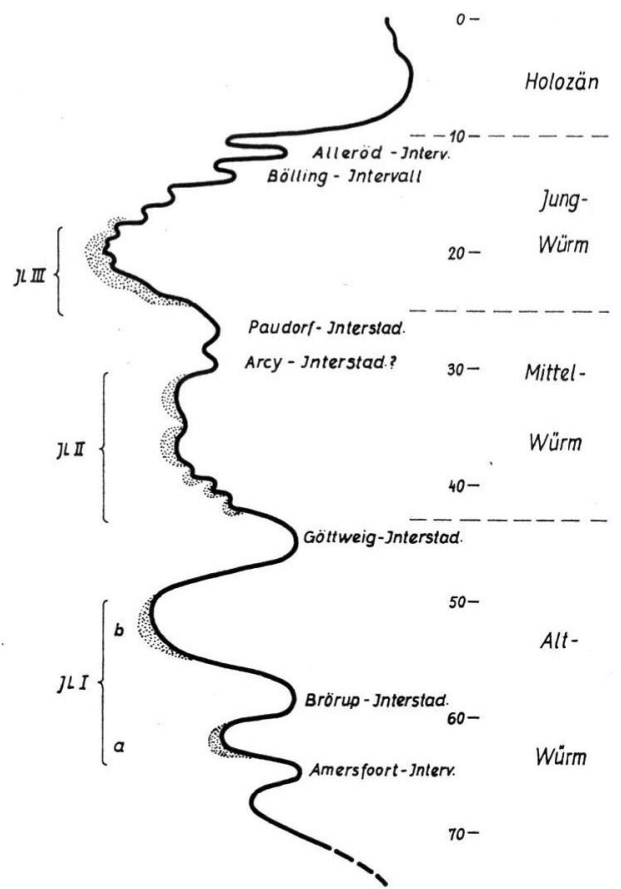

Abb. 8. Schematische Kurve, den Ablauf der Letzten Eiszeit in Mitteleuropa darstellend. Sie ist weder eine reine Temperatur- noch eine Eisrand-Kurve - für beides reichen die Beobachtungen noch nicht aus - sondern mehr ein Schaubild. Die feine Punktierung deutet die Hauptzeiten der Lößbildung an.

Interstadials bis über den Maximal-Vorstoß hinaus umfassen sollte. Aber die Grenzziehung vom Mittel- zum Spät-Würm ist unbefriedigend, und das Wesentliche der WürmEiszeit, die Gliederung in zwei Hauptvorstöße und eine längere dazwischenliegende Zeit, kommt nicht zum Ausdruck. So erscheint die Einteilung in Alt-, Mittel- und Jungwürm als besser, selbst auf die Gefahr hin, daß J u n g würm mit S pä twürm (im Sinne von "Spätglazial“) verwechselt wird.

Dabei ist die Grenzziehung zwischen den einzelnen Abschnitten noch kurz zu erörtern. Wenn man die Profile im Gelände sieht, etwa ein Lößprofil, so liegt es nahe, eine Grenze dort zu legen, wo über einem fossilen Boden ein neuer kaltzeitlicher Abschnitt beginnt. An dieser Stelle ist meist eine scharfe Grenze vorhanden, während die fossile Bodenbildung nach unten hin meist allmählich verläuft. Die Grenze: Letzte Interglazialzeit/Würm kann man überhaupt nur so ziehen, d.h. am Ende einer W a r m zeit. Will man nun weiter im Würm die Grenzen entspechend legen, so müßte die Grenze Alt/ Mittel-Würm unmittelbar ü b e $r$ der Göttweiger Verlehmung, die Grenze Mittel/JungWürm oberhalb der Paudorfer Verlehmung gezogen werden. (Die Folgerung, daß man dann die postg la zi a le Bodenbildung noch zum Jung w ü r m rechnen müßte, d.h. daß die Grenze Würm/Postglazial überhaupt verschwinden würde, kann man natürlich nicht ziehen; hier muß die Grenze am Ende einer $\mathrm{K}$ a $1 \mathrm{t}$ zeit gezogen werden.)

Würde man innerhalb der Würm-Eiszeit die Grenzen jeweils an das Ende der einzelnen Kaltzeiten legen, so müßten sie v o r Beginn des Göttweiger Interstadials, d. h. etwa bei 48000 J.v.h., und v o r Beginn des Arcy- und Paudorf-Interstadials, d. h. bei 3132000 gezogen werden. Das befriedigt aber nicht und hat den Nachteil, daß in einem Profil (z. B. im Löß) die Bodenbildung zu einem jüngeren Abschnitt, das Substrat, in dem sich der Boden gebildet hat, aber zu einem älteren gehört. So wird man also das Altwürm mit der Göttweiger Verlehmung (d. h. nach dem heutigen Stande der Kenntnisse bei rund 43000 J.v.h.), das Mittelwürm mit der Paudorfer Verlehmung enden lassen. Daß diese 
Abgrenzung in anderen Fällen wieder Schwierigkeiten mit sich bringt, ist sicher. Aber jede andere Abgrenzung bringt wieder neue Schwierigkeiten.

Die hier vorgeschlagene Abgrenzung würde auch einigermaßen den prähistorischen Gegebenheiten in Mitteleuropa gerecht werden, insofern als das Altwürm im wesentlichen mit dem Auftreten der Moustier-Kulturen, das Mittelwürm mit dem Aurignac (im weiteren Sinne, d.h. mit Châtelperron usw.), das Jungwürm mit dem Gravette (+Solutré) und Magdalen zusammenfallen würde.

\section{Vergleich des Ablaufs der Letzten Eiszeit in Nordamerika und Europa}

Wenn im einzelnen auch noch manche Frage offen bleibt, so ergibt sich doch eine weitgehende Gleichartigkeit des Ablaufes der Letzten Eiszeit in den beiden Kontinenten. Dieser Ablauf ist in einzelnen Phasen sogar erstaunlich gleichartig - man denke nur an die Gleichzeitigkeit des Alleröd- mit dem Two-Creeks-Intervall und des darauf folgenden Salpausselkä-Vorstoßes mit dem Valders-Vorstoß. Wir dürfen danach annehmen, daß auch die anderen größeren Interstadiale und Vorstöße im Großen und Ganzen gleichzeitig waren, d. h. auf überregionale klimatische Schwankungen zurückgehen.

Tatsächlich zeigt sich, daß wir in beiden Kontinenten zwei große Eisvorstöße während der Letzten Eiszeit gehabt haben, einen älteren und einen jüngeren. Dabei ist in fast allen Gebieten der jüngere Vorstoß der weitergehende, der ältere der weniger weite. Das gilt für die nordeuropäische Vergletscherung anscheinend ohne jede Ausnahme, für das alpine Gebiet, soweit mir bekannt, ebenfalls. Auch in Nordamerika war im größten Teil Teil des Bereiches der jüngere Vorstoß offenbar der weiterreichende. Doch wird in Ohio eine etwa gleichweite Ausdehnung des früheren Vorstoßes angenommen. Auch das Iowan stellt möglicherweise eine frühere weitere Ausbreitung des älteren Vorstoßes in diesem besonderen Gebiet dar.

Die beiden Vorstöße waren getrennt durch einen mittleren Abschnitt von wechselndem klimatischem Charakter. Der von $\mathrm{H}$. Gross für diesen Abschnitt vorgeschlagene Name "Göttweiger Interstadialkomplex" erscheint nicht ganz zutreffend. Denn es handelt sich offenbar um eine Zeit wechselnden Klimacharakters. Wärmere Zeiten, wie vor allem das eigentliche Göttweiger Interstadial (bei rund 45000 J.v.h.), ferner das (noch fragliche) Arcy- und das Paudorfer Interstadial wechselten ab mit Zeiten der Lößbildung, die wir sowohl in Nordamerika (Roxana-Löß, 35-37000 J.v.h.) wie in Europa (Löß von Fellabrunn bei $32000,42500 \mathrm{~J}$. usw.) hatten. Man kann diese m. E. nicht einfach als Lößbildungen in trockeneren Zeiten eines Interstadials auffassen, sondern muß in ihnen bis zum Beweise des Gegenteils normale kaltzeitliche Lösse sehen. Auch die Pollenanalysen von A. Leroi-Gourhan (1959) weisen auf einen Wechsel kälterer und wärmerer Zeiten. So wird man diesen Abschnitt am besten einfach als Mittelwü rm bzw. Mittelwisconsin bezeichnen. Im einzelnen dürfte das Port-Talbot-Interstadial dem Göttweiger Interstadial (im engeren Sinne), das Plum-Point- bzw. Farmdale-Interstadial dem Arcyund Paudorfer Interstadial entsprechen.

Trifft dies zu, dann würde die Hauptfundschicht der Salzofenhöhle (mit $34000 \pm$ 3000 J.) gerade in eine kältere Zeit fallen. Die Bestimmung könnte entweder um rund 10000 Jahre zu jung sein und die Fundschicht tatsächlich in das echte Göttweiger Interstadial gehören, oder aber die Bestimmung müßte etwas zu alt sein und die Fundschicht mit dem Arcy-Interstadial zusammenfallen. In dieses gehört, wie schon gesagt wurde, wahrscheinlich die Olschewa-Kultur der Potočka-Höhle.

Nehmen wir als sicher an, daß in Europa und im östlichen Nordamerika ein größeres Interstadial (Talbot, Göttweig) zwischen 50 und $40000 \mathrm{~J}$. gelegen hat, so ist, wie schon oben betont wurde, bisher keine befriedigende Übereinstimmung mit den Werten des westlichen Nordamerikas vorhanden. So bleibt im einzelnen natürlich noch sehr viel zu tun, bis wir eine sichere Parallelisierung zwischen Amerika und Europa und bis wir über- 
haupt ein klares Bild über den Ablauf der Letzten Eiszeit haben. Exakte C14-Bestimmungen in möglichst großer Zahl sind hier das dringendste Erfordernis.

\section{Weitere Bemerkungen zur Gliederung der Würm-Kaltzeit}

Mit der "Gliederung der Würmkaltzeit" hat sich in einer besonderen Schrift neuerdings nochmals J. BüDEL (1960) beschäftigt und ist dabei zu dem Ergebnis gekommen, seine Auffassung einer eirheitlichen Würmkaltzeit, die er im Wesentlichen schon 1953 aufgestellt habe, sei die richtige und die von mir gegebenen Kurven, die ich allmählich verbessert und dem jeweiligen Stande der Wissenschaft anzugleichen versucht habe, seien nicht zutreffend

BüDEL gibt aus seiner früheren Arbeit nur die Abb. 2 nochmals wieder und betont, daß er diese im großen und ganzen aufrecht erhalte. In seiner früheren Arbeit gab er aber eine zweite Kurve (Abb. 4), an der ich 1954 (S. 45) etwas Kritik übte. Die „Letzte Warmzeit“, die er darin unterscheidet, liegt nach ihm zwischen der "Jungriß-Kaltzeit" und der „Würm-Kaltzeit“, und sie sei durch eine Elephas-primigenius-Fauna charakterisiert. Das Moustérien habe während des Vorletzten Interglazials und der Jungriß-Kaltzeit, das Aurignacien und Magdalénien während der Würm-Kaltzeit bestanden.

BüDEL kommt jetzt zu dem Ergebnis, sein damals angenommenes „Letztes Interglazial“ sei nun also die Eem-Zeit, und darauf folge seine einheitliche' Würm-Kaltzeit. So einfach ist aber die Sache nicht. Denn das Moustérien, die Kultur des Neandertalers, beginnt, wie ich 1954 bereits betonte, in der zweiten Hälfte des Eem-Interglazials (das im übrigen durch Elephas antiquns charakterisiert ist) und geht bis etwa zur Mitte der WürmKaltzeit. Was BüDEL in Abb. 4 als „Letztes Interglazial“ verzeichnet, das ist in etwa das große Würm-Interstadial, das sowohl in Nordamerika wie in Europa festgestellt worden ist (das aber natürlich in einer Klimakurve etwas anders aussehen muß, als auf BüDELS Abb. 4 dargestellt ist).

Folgende Tatsachen müssen bei der Rekonstruktion einer Kurve der Letzten Vereisung berücksichtigt werden:

1. Das letzte echte Interglazial mit Meereshochstand ("Monastir") war das EemInterglazial (Sangamon-Interglazial in Nordamerika) mit seinem charakteristischen Pollendiagramm, das in Hunderten von Profilen in West-, Mittel- und Osteuropa nachgewiesen ist. Es bildete einen Leithorizont, der auch in den Alpen viel mehr als Basis weiterer Untersuchungen verwendet werden sollte, als dies bisher geschehen ist. Das Ende dieses Interglazials scheint etwa 70-80000 Jahre zurïck zu liegen.

2. A. Penck wollte, wenn man seine Ausführungen sinngemäß liest, als Würm alles aufgefaßt wissen, was nach der letzten echten Interglazialzeit liegt. Das war die Interglazialzeit von Großweil-Ohlsdorf, Uznach usw., d.h. die Eem-Interglazialzeit. Wenn er später die zuerst von ihm innerhalb der Würm-Eiszeit angenommenen großen Schwankungen (Laufenschwankung, Achenschwankung) aufgegeben hat, so ändert das an dieser Grundauffassung nichts; auch nicht, wenn er in einzelnen Gebieten etwas als Würm bezeichnet hat, was Riß ist, oder als Riß, was Würm ist - PENCK konnte schließlich auch einmal irren.

3. Die Würm-Kaltzeit begann mit allmählich absteigenden Temperatur-Schwankungen, wie ich sie in meinen verschiedenen Kurven angedeutet habe. ${ }^{5}$ ) Dies war schon durch die Untersuchungen von Helga REICH (1953) über die Schieferkohlen von GroßweilOhlsdorf wahrscheinlich gemacht worden und ist inzwischen durch den Nachweis der

5) In einem Punkte seiner Kritik hat J. BüDEL zweifellos recht. Ich hätte die (dick ausgezogene) Kurve des Eisrandes nicht voll ausziehen, sondern unterbrechen müssen. Aber ich dachte, die Bezeichnung „schematische Kurve" und der Zusatz, daß die „Lage des Eisrandes für den größten Teil der Kurve nur vermutet" sei, genüge. Anscheinend ist das aber doch nicht genügend gewesen. 
Interstadiale (bzw. Intervalle) von St. Pierre, Amersfoort, Brörup und Loopstedt bestätigt worden.

4. Überall, wo das sog. „Kalte Moustérien“ geologisch datiert werden kann, gehört es ohne jede Ausnahme in die Kaltzei $n$ a c h dem Eem-Interglazial. Soweit wir bisher $\mathrm{C}^{14}$ Bestimmungen haben (z. B. von Lebenstedt mit 55000 J.v.h.), bestätigen sie diese Einstufung.

5. Der erste Abschnitt der Würm-Kaltzeit war in West- und Mitteleuropa nicht nur eine Fließerdezeit, wie BüDEL annimmt, sondern auch eine e ch te Löß z e it. In Frankreich sind in dieser Zeit $\mathrm{mindestens} z$ wei echte Lösse abgelagert worden, die Moustier-Kulturen enthalten (vgl. u. a. F. Bondes 1954). Sie liegen einwandfrei über dem interglazialen Boden der Letzten Warmzeit („argile“ oder „limon“ rouge). Dasselbe gilt, wie z. Zt. laufende Untersuchungen zeigen, für das Niederrheingebiet, wo diese LößHorizonte auf der den R/W-interglazialen Boden tragenden Unteren Mittelterrasse (Riß) entwickelt sind. Der Ablauf: Fließerde - Lößzeit gilt, wie ich in meiner 1956 gegebenen Kurve angedeutet habe, für jede einzelne Phase, n icht für die Würmzeit als $\mathrm{Ganzes}$ (wenn auch die Lößbildung in ihrem $\mathrm{Um} \mathrm{m}$ a $\mathrm{ng}$ vielleicht zugenommen hat).

6. Sowohl in Nordamerika wie in Europa folgte auf den ersten Würm-Abschnitt eine längere Zeit von wechselndem klimatischem Charakter mit wahrscheinlich mehreren Schwankungen, die im einzelnen noch genauer zu untersuchen sind. Wie will man ohne eine länger dauernde Erwärmung die Begehung der Salzofenhöhle in $2000 \mathrm{~m}$ Höhe oder die Besiedelung der Potočkahöhle im Olschewien vor wahrscheinlich mehr als 30000 Jahren sonst erklären?

7. Der zweite große Vorstoß des Würm-Eises begann in Europa wie in Nordamerika etwa um 25000 J.v.h. und führte bis zur Maximalausdehnung. Diese ist nicht synchron, sondern "metachron" und liegt in den verschiedenen Gebieten Nordeuropas und Nordamerikas zwischen 20000 und 16000 J.v.h.

Um rund 10000 J.v.h. setzte die entscheidende Klimabesserung ein, die das Holozän einleitete.Hier handelt es sich um ein weltweites Ereignis, das am ehesten geeignet ist, die Grundlage für die Grenze Pleistozän/Holozän zu liefern.

Zum Schluß sei nochmals betont, daß auch die jetzt gegebene Darstellung vom Ablaufe der Letzten Eiszeit noch höchst hypothetisch und wenig befriedigend ist. Sie kann nur versuchen, den heutigen Stand der Kenntnisse wiederzugeben, der nicht nur sehr lückenhaft, sondern z. T. in sich geradezu widersprüchlich ist. Aber erst bei einem Versuch, das Fazit aus den bisherigen Feststellungen zu ziehen, zeigen sich die Lüicken und Widersprüche. Hier haben neue Untersuchungen und neue $C^{14}$-Bestimmungen einzusetzen.

\section{$\mathrm{Schriften-Nachweis}$}

Andersen, Sv. Th., De Vries, Hl. \& Zagwijn, W. H.: Climatic change and radiocarbon dating in the Weichselian Glacial of Denmark and the Netherlands. - Geol. en Mijnbouw 39 (n. s. 22), S. 38-42, 1960.

BAyER, Josef: Der Mensch im Eiszeitalter. - Wien 1917.

Bordes, Fr.: Les limons quaternaires du Bassin de la Seine. - Arch. Inst. Paléont. Humaine, Mém. 26, Paris 1954.

BRAndtner, Fr.: Jungpleistozäner Löß und fossi'e Böden in Niederösterreich. - Eisz. u. Geg. 4/5, S. 49-82, Óhringen 1954. - - Lößstratigraphie und paläolithische Kulturabfolge in Niederösterreich und in den angrenzenden Gebieten. - Ebendort 7, S. 127-175, 1956.

Brodar, S.: Das Paläolithikum in Jugoslawien. - Quartär 1, S. 140-172, Berlin 1938.

Broecker, W. S. \& OrR, Ph. C.: Radiocarbon chronology of Lake Lahontan and Lake Bonneville. - Bull. geol. Soc. Amer. 69, S. 1009-1032, 1958.

BüDEL, Julius: Die „periglazial“-morpho'ogischen Wirkungen des Eiszeitklimas auf der ganzen Erde. - Erdkunde 7, S. 249-266, Bonn 1953. - - Die Gliederung der Würmkaltzeit. Würzburger geogr. Arb., Heft 8, 45 S., Würzburg 1960. 
Burns, G. W.: Wisconsin age forests in western Ohio II. Vegetation and burial conditions. Ohio J. Sci. 58, S. 220-230, Columbus 1958.

Coleman, A. P.: The Pleistocene of the Toronto Region. - Ontario Departm. of Mines, Ann. Rep. 41, Pt. VII, 69 S., Toronto 1933.

Dreimanis, A.: Proposed local stratigraphy of the Wisconsin glacial stage in the area south of London, Southwestern Ontario. - Contrib. Departm. Geology, Univ. West. Ontario, Nr. 25, S. 24-30, London / Canada 1959.

Ebers, Edith: Hauptwürm, Spätwürm, Frühwürm und die Frage der älteren Würmschotter. Eisz. u. Geg. 6, S. 96-109, 1955. - - Drumlinkerne, ältere Würmschotter und das WürmInterstadial-Profil von Hörmating/Obb. - Dieser Band, S. 64-76, 1960.

Emiliani, C.: Paleotemperature analysis of core 280 and Pleistocene correlations. - Journ. Geol. 66, S. 264-275, Chicago 1958.

Felgenhauer, F., Fink, J. \& DE Vries, H.: Studien zur absoluten und relativen Chronologie der fossilen Böden in Ósterreich. I. Oberfellabrunn. - Archaeologia Austriaca 25, S. 35-73, Wien 1959.

Flint, R. F.: Glacial and Pleistocene Geology. - 553 S., New York 1957.

FLINT, R. F. \& GALE, W. A.: Stratigraphy and radiocarbon dates at Searles Lake, California. Amer. J. Sci. 256, S. 689-714, New Haven 1958.

Flint, R. F. \& Rubin, M.: Radiocarbon dates of Pre-Mankato events in Eastern and Central North America. - Science 121, S. 649-658, 1955.

FonsYTH, Jane L.: New exposure of the buried "Sidney-type" soil a'ong upper Bush Creek, Ohio. - Bull. geol. Soc. Amer. 69, S. 1565, 1958.

Forsyth, Jane \& La Roque, A.: Age of the buried soil at Sidney, Ohio. - Bull. geol. Soc. Amer. 67, S. 1696, 1956.

Frye, J. C. \& Willman, H. B.: Classification of the Wisconsinan stage in the Lake Michigan Glacial Lobe. - Illinois State Geol. Survey, Circular 285, 16 S., Urbana/Ill. 1960.

Gawlowska, Marja: Contribution to the knowledge of the fossil flora of Cimoszkowicze (poln., engl.). - Ann. Soc. géol. Pologne 10, S. 1-29, Krakau 1934.

Goldthwait, R. P.: Wisconsin age forests in western Ohio I. Age and glacial events. - Ohio Journ. Sci. 58, S. 209-216, Columbus 1958. - - Scenes in Ohio during the last ice age. Ebendort 59, S. 193-216, Columbus 1959.

Gross, H.: Das Göttweiger Interstadial, ein zweiter Leithorizont der letzten Vereisung. - Dieses Jahrb. 7, S. 87-101, 1956. - - Die bisherigen Ergebnisse von C14-Messungen und paläontologischen Untersuchungen usw. - Dieses Jahrb. 9, S. 155-187, 1958.

Haring, A., DE VRIES, A. E. \& DE VRIes, H.: Radiocarbon Dating up to 70000 Years by Isotopic Enrichment. - Science 128, S. 472/473, 1958.

Leighton, M. M.: A notable type Pleistocene section - the Farm Creek exposure near Peoria, Illinois. - Journ. Geol. 34, S. 167-174, Chicago 1926. - - The classification of the Wisconsin Glacial Stage of the Central Region of the United States. - Journ. Geol. 68, Chicago 1960 (im Druck).

Leroi-Gourhan, Arlette: Communication au Congrès de la Societé Préhistorique Francaise, Monaco, Août 1959 (Temperatur- und Feuchtigkeitskurven für die letzten 50000 Jahre).

Lütrig, Gerd: Eiszeit - Stadium - Phase - Staffel. - Geol. Jb. 76, S. 235-260, Hannover 1959.

Movius, H. L., Jr.: Radiocarbon Dates and Upper Palaeolithic Archaeology. - Current Anthrop., 1, S. 355-391, Chicago 1960.

NARr, K. J.: C14-Daten und die Gliederung des Jungpleistozäns. - Forsch. u. Fortschr. 33, S. 147 bis 151, Berlin 1959. - - Nochmals: C14-Daten und die Gliederung des Jungpleistozäns. Ebendort 34, S. 90/91, 1960.

Oeschger, H., Schwarz, U., Gfeller, Chr.: Bern Radiocarbon Dates I. - Amer. J. Sci., Radiocarbon Supplement 1, S. 133-143, New Haven 1959.

ProšEK, Fr. \& LožEK, V.: Stratigraphische Übersicht des tschechoslowakischen Quartärs. - Dieses Jahrb. 8, S. 37-90, 1957.

ReICH, Helga: Die Vegetationsentwicklung der Interglaziale von Großweil-Ohlstadt und Pfefferbichl im Bayrischen Alpenvorland. - F!ora 140, S. 386-443, Jena 1953.

REMr, Horst: Der Löß am unteren Mittel- und Niederrhein. - Dieses Jahrb. 11, 1960.

SchönhaLs, E.: Ưber fossile Böden im nichtvereisten Gebiet. - Dieses Jahrb. 1, S. 109-130, 1951.

Soergel, W.: Lösse, Eiszeiten und paläolithische Kulturen. - 177 S., Jena (G. Fischer) 1919.

Schмid, Elisabeth: Von den Sedimenten der Salzofenhöhle. - Sber. österr. Akad. Wiss., math. nat. K1. I, 166, S. 43-55, Wien 1957.

Terasmae, J.: Non-glacial Deposits in the St. Lawrence Lowlands, Quebec. - Geol. Survey Canada, Bull. 46, S. 13-28, Ottawa 1958. - - A Palynological Study of Pleistocene Interglacial Beds at Toronto, Ontario. - Geol. Survey Canada, Bull. 56, S. 23-40, Ottawa 1960. 
DE VRIES, Hl.: Radiocarbon Dates for upper Eem and Würm-interstadial samples. - Dieses Jahrb. 9, S. 10-17, 1958. - Radiocarbon dating of the fossile soils at Ober Fellabrunn. - Proc. kon. ned. Akad. Wet. Amsterdam (B) 62, S. 84-91, 1959.

Woldstedt, P.: Das Eiszeitalter, 1. Aufl., Stuttgart 1929 (2. Aufl. 1954). - - Saaleeiszeit, Warthestadium und Weichseleiszeit in Norddeutschland. - Dieses Jahrb. 4/5, S. 34-48, 1954. - Über die Gliederung der Würm-Eiszeit und die Stellung der Lösse in ihr. - Dieses Jahrb. 7, S. 78-86, 1956. - - Eine neue Kurve der Würm-Eiszeit. - Dieses Jahrb. 9, S. 151-154, 1958 (1958a). - - Das Eiszeitalter, 2. Aufl., Bd. II, 438 S., Stuttgart 1958 (1958b). - - Alte Strandlinien des Pleistozäns in Nordamerika und Europa. - Dieser Band, S. $12-19,1960$.

Gross, H.: Nocheinmal: Riß oder Würm? - Dieses Jb. 10, S. 65-76, 1959. - - Die Bedeutung des Göttweiger Interstadials im Ablauf der Würm-Eiszeit. - Dieser Band, S. 99-106, 1960.

Manuskr. eingeg. 22. 8. 1960.

Anschrift des Verf.: Prof. Dr. Paul Woldstedt, Bonn a. Rh., Argelanderstraße 118.

Nachtrag während des Druckes. In Abb. 8 (S. 160) habe ich den Jüngeren Löß in die Stufen JL I, JL. II und JL III gegliedert. Das steht im Widerspruch zu der früher (1956) von mir vorgeschlagenen Einteilung in JL I, JL IIa und JL IIb. Diese Einteilung lehnte sich an die frühere SoERgEssche an. Ich habe mich aber davon überzeugt, sowohl auf Grund neuerer, z. Zt. noch im Gange befindlicher Untersuchungen wie auch mündlicher Mitteilungen von E. Schönhals, daß die Einteilung in drei Stufen die bessere ist. 